\title{
Chemistry of Stars in the Sculptor Dwarf Galaxy from VLT-FLAMES
}

\author{
Kim A. Venn ${ }^{1,2}$ and V. Hill ${ }^{3}$ \\ ${ }^{1}$ Macalester College, 1600 Grand Avenue, Saint Paul, MN, 55105 \\ ${ }^{2}$ Department of Physics \& Astronomy, University of Victoria, Victoria, Canada \\ email: venn@macalester.edu \\ ${ }^{3}$ GEPI, Observatoire de Meudon, CNRS UMR 8111, France \\ email: Vanessa.Hill@obspm.fr
}

\begin{abstract}
The chemical composition of 91 stars in the Sculptor dwarf spheroidal galaxy is presented as determined from spectra taken with the FLAMES multiobject spectrograph in the Medusa mode. The analysis methods are outlined. The $[\alpha / \mathrm{Fe}]$ ratios are shown for $\mathrm{Mg}$, $\mathrm{Ca}$, and $\mathrm{Ti}$, and compared with those of Galactic stars. Heavy element abundance ratios (Y, Ba, and $\mathrm{Eu})$ are also presented. Since the Sculptor dwarf galaxy has had a significantly different star formation history and chemical evolution than the Galaxy, then comparison of Sculptor's metalpoor (old) stars to similar metallicity stars in the Galaxy can be used to discuss galaxy formation scenarios, as well as test some of our fundamental assumptions in stellar nucleosynthesis.
\end{abstract}

Keywords. Stars: abundances, Galaxy: abundances, formation, evolution, galaxies: abundances, dwarf, evolution, Local Group

\section{Introduction}

Simulations of the formation of the Galactic halo (e.g., Font et al. 2005, Bullock et al. 2002, Johnston et al. 2002) suggest that the field halo stars originated in small dwarf galaxies that were tidally disrupted and accreted into the halo. These stars should have kinematics with similar phase space, and a coherent chemical evolution related to the star formation history that the dwarf galaxy underwent before merger. Other simulations of the Galactic thick disk also suggest formation through the merger of a dwarf galaxy (though possibly larger than those in the Galactic halo, i.e., LMC-sized, and/or gas-rich dwarfs; e.g., Abadi et al. 2003, Navarro et al. 2004, Governato et al. 2004, Brook et al. 2004, Helmi et al. 2005).

A simple test of these formation scenarios is a comparison of the stellar abundances in the Local Group dwarf galaxies. The Local Group dwarfs show a wide range in masses, gas contents, and star formation histories. Analysis of the chemistries of the old, metal-poor stars in these dwarf galaxies should have chemical properties in common with old, metalpoor stars in the Galactic halo and/or thick disk, particularly if merging is continuous until redshifts of $\mathrm{z}=1-2$.

The nearby dwarf spheroidals and the Magellanic Clouds are the only Local Group dwarf galaxies for which the old, metal-poor stars on the red giant branch are bright enough for high $\mathrm{S} / \mathrm{N}$, high resolution spectroscopic analyses. As part of the French GTO program on high resolution abundances in the Sculptor dSph galaxy, we have used the FLAMES multi-object spectrograph at the VLT to observe red giants in the Sculptor dwarf galaxy. 


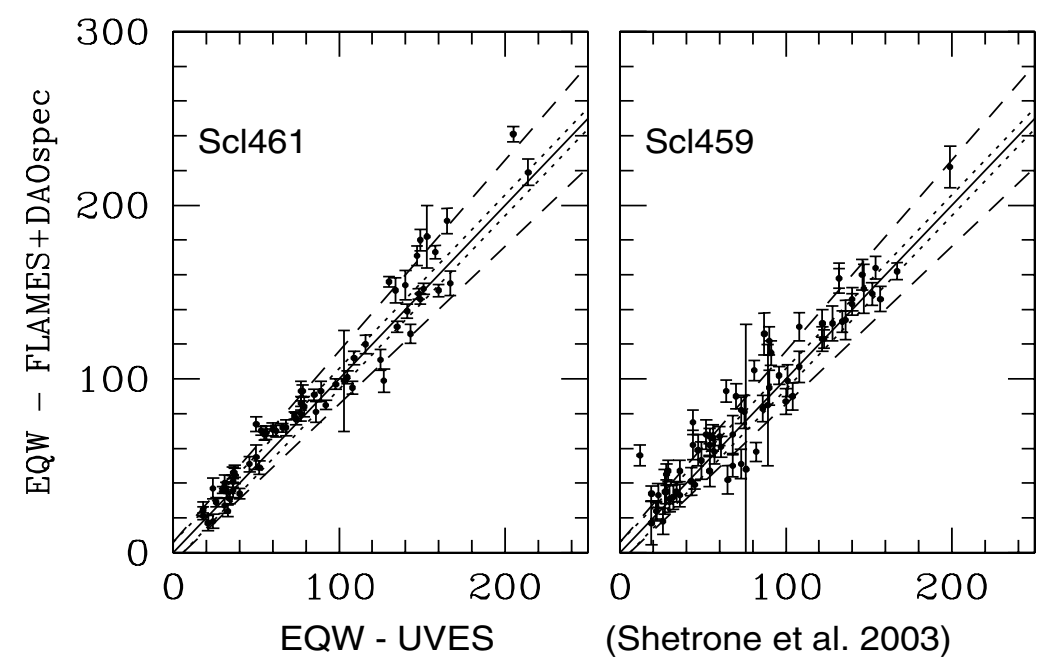

Figure 1. Spectral line equivalent widths measured from the FLAMES Medusa-fiber spectra using DAOspec. These values are compared to those measured by Shetrone et al. (2003) using splot in IRAF to measure an integrated area from higher resolution UVES spectra. Solid line is the one-to-one relationship, dotted lines represent $\pm 6 \mathrm{~m} \AA$ error, and dashed lines show this minimum error plus a $10 \%$ x EQW uncertainty.

\section{Method}

VLT/FLAMES observations were made in Medusa mode using 117 fibers placed on stars with $\mathrm{I} \geqslant 17.5$ over a 25 arcmin diameter field of view. Combined signal-to-noise ratios were $\geqslant 30$ which is adequate for a model atmospheres analysis, with the exception of 20 stars which reached to only $\sim 25$. Four wavelength setups that covered $\sim 20 \mathrm{~nm}$ each were selected to optimize the number of iron features and observe the critical $\alpha$-element and heavy element features. These included HR10, HR13, HR14, and HR15, which have central wavelengths of $548.8,627.3,651.5$, and $679.7 \mathrm{~nm}$, respectively.

The FLAMES data were reduced, extracted and wavelength calibrated using the GIRBLDRS pipeline provided by the FLAMES consortium (Geneva Observatory, Blecha \& Simond 2004). For velocity and equivalent width measurements, we used DAOspec (Stetson \& Pancino 2005). Because DAOspec is a Gaussian line fitting routine, we have examined the zero point accuracy for both velocity and equivalent width measurements by comparing with independent previous measurements from higher resolution UVES spectroscopy for stars in Sculptor that we deliberately reobserved. In Fig. 1, we show line equivalent width measurements from DAOspec, which are in fair to good agreement with the UVES measurements. The line measurements are expected to deviate from Gaussian profiles primarily for stronger lines where DAOspec would fail to accurately measure the Doppler broadened line wings, however we do not find a significant offset for our comparison stars, particularly within the DAOspec fitting errors shown by the errorbars in Fig. 1.

Initial estimates for the effective temperature and gravity are based on (V-I) and (V-K) photometry. Initial temperatures were checked by plotting FeI vs excitation potential; any stars with slope $\geqslant 2 \sigma$ from the mean was re-examined. Initial (physical) gravity estimates were adjusted such that the same abundance of iron was determined from neutral and 
ionized iron line species. Microturbulent velocities were determined by minimizing the slope between FeI line abundances and their equivalent width measurments.

Model atmospheres were taken from the computations of the OSMARCS code (Plez, Brett, \& Nordlund 1992, Edvardsson et al. 1993, Asplund et al. 1997). Elemental abundances are from the Spite suite of LTE abundance calculation codes. No corrections have been made to the abundances for non-LTE effects. For the results presented in this proceedings, no hyperfine structure corrections have been applied either.

\section{Results}

Elemental abundances for 91 (85 new) stars in the Sculptor dwarf spheroidal galaxy have been determined from the FLAMES/GIRAFFE spectroscopy. In this proceedings, we present results for $\alpha$-elements $(\mathrm{Mg}, \mathrm{Ca}, \mathrm{Ti})$ and heavy elements (Y, Ba, and $\mathrm{Eu}$ ).

\subsection{The $[\alpha / \mathrm{Fe}]$ ratios}

It is clear in Fig. 2 that the $[\mathrm{Mg} / \mathrm{Fe}],[\mathrm{Ca} / \mathrm{Fe}]$, and $[\mathrm{Ti} / \mathrm{Fe}]$ results present a consistent picture for the chemical evolution of the $\alpha$-elements in Sculptor and that picture differs from that for the Galactic comparison stars. The chemistry for the Galactic comparison stars is from the compilation by Venn et al.(2004; small black dots; we note that most of the comparison stars in the intermediate-metallicity regime are from Fulbright 2000). Previously published VLT+UVES spectroscopic results for red giants in Sculptor (Shetrone et al.2003, large solid circles; Geisler et al.2005, solid triangles) and globular cluster comparison stars (Shetrone et al.2003, large black squares) are shown (all with errorbars) for comparison.

The new FLAMES spectroscopic results are in excellent agreement with the trends seen in the previously published (9) Sculptor stars. This is an important first result since the resolution of the MEDUSA fiber spectra is about a factor of two lower than UVES. The new FLAMES results also extend the metallicity range sampled in Sculptor by a factor of two, down to $[\mathrm{Fe} / \mathrm{H}]=-2.4$, and help to confirm that the most metalpoor stars in Sculptor have Galactic halo-like $[\alpha / \mathrm{Fe}]$ abundance ratios. At a metallicity of $[\mathrm{Fe} / \mathrm{H}]=-1.8$, the $[\alpha / \mathrm{Fe}]$ ratios begin to decrease towards subsolar $[\alpha / \mathrm{Fe}]$ ratios at metallicities of $[\mathrm{Fe} / \mathrm{H}]=-1.0$. The slope in the $[\mathrm{Mg} / \mathrm{Fe}]$ ratios may be slightly steeper than in $[\mathrm{Ca} / \mathrm{Fe}]$ (and possibly $[\mathrm{Ti} / \mathrm{Fe}]$ ), however the scatter in the $[\mathrm{Mg} / \mathrm{Fe}]$ ratios is also larger than in $[\mathrm{Ca} / \mathrm{Fe}]$.

\subsection{The heavy element ratios}

Even though we have not yet applied hyperfine corrections to the YII, BaII, nor EuII elemental abundances (thus the ' $n$ ' to the y-axes label in Fig. 3), these corrections are typically small in the red giants in this sample, e.g., Shetrone et al.(2003) found hfs corrections $\leqslant 0.2$ dex in the UVES spectral analyses. Thus, examination of our preliminary heavy element abundance ratios in Fig. 3 are warranted.

The $[\mathrm{Eu} / \mathrm{Fe}]$ results from the FLAMES spectroscopic analyses are in very good agreement with the published results from Shetrone et al.(2003) and Geisler et al.(2005). The $[\mathrm{Eu} / \mathrm{Fe}]$ ratios in the new stars follow a similar trend as the published UVES results. The slope is similar to that of the $[\alpha / \mathrm{Fe}]$ ratios although with much larger scatter. The scatter is most likely due to our analysis, which is limited to only one weak line of EuII and thus subject to uncertainties in the $\mathrm{S} / \mathrm{N}$ ratios of the spectra and setting of the continuum level. It is clear that the Sculptor $[\mathrm{Eu} / \mathrm{Fe}]$ ratios do not resemble those of the stars in the Galaxy. In the most metal-poor stars, $[\mathrm{Eu} / \mathrm{Fe}]$ appears to be higher than the Galactic comparison halo stars, including the Galactic globular cluster comparison stars. 

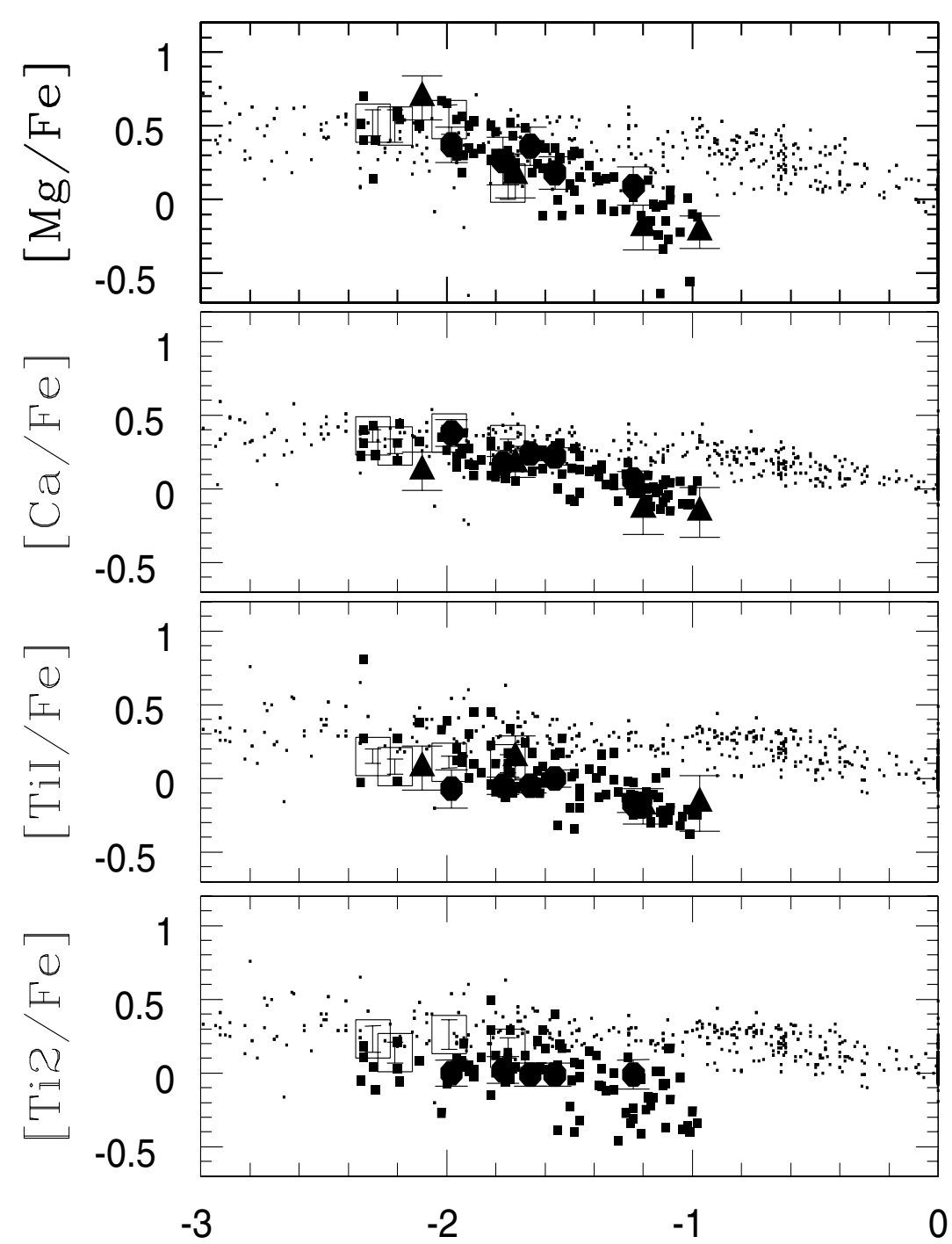

$[\mathrm{Fel} / \mathrm{H}]$

Figure 2. Ratio of the $\alpha$-element abundances in the Sculptor stars with respect to iron. Published data based on UVES spectroscopy is shown by large solid symbols (see text). The new FLAMES spectroscopic results are shown as small, solid squares. For comparison, Galactic abundances are shown by dots from the compilation by Venn et al. (2004).

Amongst the more metal-rich Sculptor stars, the [Eu/Fe] ratios are clearly lower than the Galactic comparisons (in fact, sub-solar). The similarity in the $[\mathrm{Eu} / \mathrm{Fe}]$ trend to the $[\alpha / \mathrm{Fe}]$ trend strongly suggests a similar nucleosynthetic source. Since europium is known to be primarily formed by the r-process (95\% from the r-process in the solar composition, Burris et al. 2000), which is linked to the massive stars that produce $\alpha$ elements (at least in some models, e.g., Duncan et al. 1986, Woosley et al. 1994), then the decreasing trend in $[\mathrm{Eu} / \mathrm{Fe}]$ and in $[\alpha / \mathrm{Fe}]$ is expected since Sculptor has had no significant star formation after the first epoch (Tolstoy et al. 2003). 


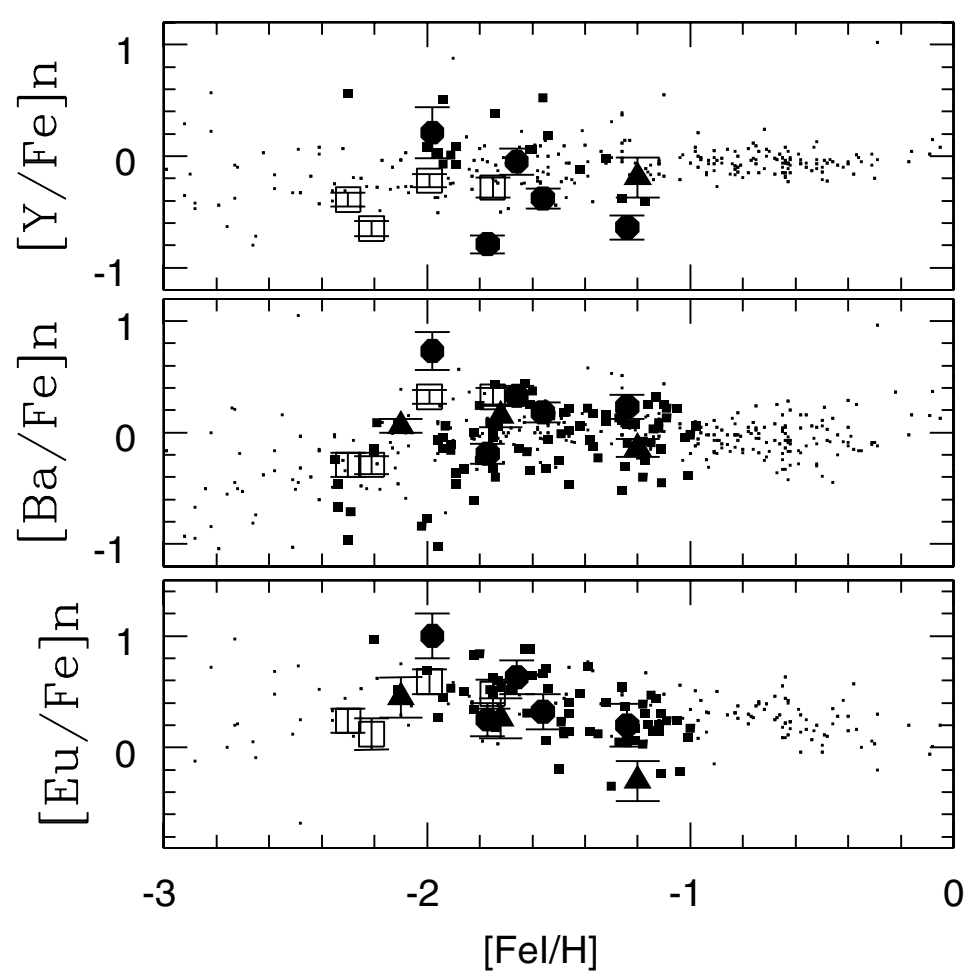

Figure 3. Ratio of the heavy-element abundances in the Sculptor stars with respect to iron. Published data based on UVES spectroscopy is shown by large solid symbols (see text). The new FLAMES spectroscopic results are shown as small, solid squares. For comparison, Galactic abundances are shown by dots from the compilation by Venn et al. (2004).

The barium and yttrium ratios tell another story. The rise in the $[\mathrm{Ba} / \mathrm{Fe}]$ ratios in metal-poor stars in the Galaxy is interpreted as evidence for the rise in the s-process contributions to Ba from AGB stars (e.g., Busso et al. 1999, although Ba can also form through rapid neutron captures). The new $[\mathrm{Ba} / \mathrm{Fe}]$ results from the FLAMES spectroscopy is exciting because we can see the rise in the s-process in the Sculptor stars now; previously, from only the UVES data, the $[\mathrm{Ba} / \mathrm{Fe}]$ ratios seemed flat which could lead to different nucleosynthetic interpretations. The $[\mathrm{Y} / \mathrm{Fe}]$ results are also exciting; even though we have determined YII abundances from over 20 stars in Sculptor, it is still difficult to interpret the $[\mathrm{Y} / \mathrm{Fe}]$ ratios because it is not clear whether $[\mathrm{Y} / \mathrm{Fe}]$ shows a large scatter or a downward trend with metallicity. If the former, then this suggests multiple sites for the production of yttrium (and not strongly linked to the rise of the s-process as seen in barium). If the latter, then the yttrium production appears to be closely linked with the r-process site that produces europium.

\section{Conclusions}

We present results from our analysis of FLAMES spectroscopic observations of red giant branch stars in the Sculptor dwarf galaxy. While the $[\alpha / \mathrm{Fe}]$ ratios decrease with metallicity as expected (given Sculptor's star formation history), we find that [Eu/Fe] also appears to decrease smoothly. This is interpreted as the r-process site for Eu being linked to the site of $\alpha$-element production. We see a rise in the $[\mathrm{Ba} / \mathrm{Fe}]$ ratios indicative 
of barium production by the main s-process in AGB stars, which had not been clearly seen before in Sculptor. It is not clear whether $[\mathrm{Y} / \mathrm{Fe}]$ is decreasing (like $[\mathrm{Eu} / \mathrm{Fe}]$, thus primarily r-process production) or whether $[\mathrm{Y} / \mathrm{Fe}]$ shows a large scatter suggestive of multiple nucleosynthetic sites. As we finalize our analysis of the chemical composition of these old, metal-poor stars, then we expect to provide new constraints for fundamental tests of stellar nucleosynthesis.

Our preliminary results show that most of the stars in the Sculptor dwarf galaxy do not have similar chemical abundance ratios to stars in the Galaxy. This is unlike the predictions from hierarchical galaxy formation models, unless Sculptor (and its old stars) is not typical of the dwarf galaxies that merged to form the Milky Way. The $[\alpha / \mathrm{Fe}]$ ratios suggest the most metal-poor stars in Sculptor $([\mathrm{Fe} / \mathrm{H}] \leqslant-1.8)$ could resemble the most metal-poor stars in the Galactic halo. However, our preliminary results for the new $[\mathrm{Y} / \mathrm{Fe}]$ and $[\mathrm{Eu} / \mathrm{Fe}]$ appear to be higher than the Galactic halo stars, which would rule out any component of Sculptor being similar to any component of the Galaxy's stellar population.

\section{Acknowledgements}

We would like to thank DART and the French-GTO team for their support, hard work, patience, and friendship throughout this exciting project. KAV would like to thank the NSF for support through CAREER grant AST-9984073.

\section{References}

Abadi M.G., Navarro J.F., Steinmetz M., \& Eke V.R., 2003, ApJ, 597, 21

Asplund, M., Gustafsson, B., Kiselman, D., \& Eriksson, K. 1997, A\&AA, 318, 521 (erratum 323, 286)

Blecha A. \& Simond G., 2004, Doc. No. VLT-SPE-OGL-13730-0040, available at http://girbldrs.sourceforge.net

Brook C.B., Kawata D., Gibson B.K., \& Freeman K.C., 2004, ApJ, 612, 894

Bullock J.S., Wechsler R.H., \& Somerville R.S., 2002, MNRAS, 329, 246

Burris D.L., Pilachowski C.A., Armandroff T.E., Sneden C., Cowan J.J., \& Roe H., 2000, ApJ, 544,302

Busso M., Gallino R., \& Wasserburg G.J., 1999, ARA\& A, 37, 239

Duncan R.C., Shapiro S.L., \& Wasserman I., 1986, ApJ, 309, 141

Edvardsson, B., Andersen, J., Gustafsson, B., Lambert, D.L., Nissen, P.E., \& Tomkin, J. 1993, $A \mathscr{E} A, 275,101$

Font, A., Johnston K.V., Bullock J.S., \& Robertson B., 2005, ApJ submitted; astro-ph/0507114

Fulbright J.P., 2000, AJ, 120, 1841

Geisler D., Smith V.V., Wallerstein G., Gonzalez G., \& Charbonnel C., 2005, AJ, 129, 1428

Governato F., Mayer L., Wadsley J., Gardner J.P., Willman B., Hayashi E., Quinn T., Stadel J., \& Lake G., 2004, ApJ, 607, 688

Helmi A., Navarro J.F., Nordstrom B., Holmberg J., Abadi M.G., \& Steinmetz M., 2005, MN$R A S$, submitted; astro-ph/0505401

Johnston K.V., Spergel D.H., \& Haydn C., 2002, ApJ, 570, 656

Navarro J.F., Helmi A., \& Freeman K.C., 2004, ApJL, 601, 43

Plez, B., Brett, J. M., \& Nordlund, A. 1992, A\&̈A, 256, 551

Shetrone M.D., Venn K.A., Tolstoy E., Primas F., Hill V., \& Kaufer A., 2003, AJ, 125, 684

Stetson P., Pancino E., 2005, in prep.

Tolstoy E., Venn K.A., Shetrone M.D., Primas F., Hill V., Kaufer A., \& Szeifert T., 2003, AJ, 125, 707

Venn K.A., Irwin M., Shetrone M.D., Tout C.A., Hill V., \& Tolstoy E., 2004, AJ, 128, 1177

Woosley S.E., Wilson J.R., Mathews G.J., Hoffman R.D., \& Meyer B.S., 1994, ApJ, 433, 229 